\title{
Deposition of Composite Materials Using a Wire-Bar Coater for Achieving Processability and Air-Stability in Organic Field-Effect Transistors (OFETs)
}

\author{
Freddy G. Del Pozo ${ }^{\mathrm{a}, \mathrm{b}}$, Sergi Galindo ${ }^{\mathrm{a}}$, Raphael Pfattner ${ }^{\mathrm{a}}$, Concepció Rovira ${ }^{\mathrm{a}}$ and \\ Marta Mas-Torrent*a \\ ${ }^{\mathrm{a} I n s t i t u t ~ d e ~ C i e ̀ n c i a ~ d e ~ M a t e r i a l s ~ d e ~ B a r c e l o n a ~(I C M A B-C S I C) ~ a n d ~ C I B E R-B B N, ~ C a m p u s ~ U A B, ~}$ \\ Bellaterra, Barcelona, Spain 08193; \\ ${ }^{\mathrm{b}}$ Current address: Universidad Técnica de Ambato, Av. Colombia y Chile, Ambato, Tungurahua, \\ Ecuador EC180105
}

\begin{abstract}
Organic thin films based on composite materials of semiconducting dibenzo-tetrathiafulvalene (DB-TTF) and insulating styrenic matrices (Polystyrene (PS10k) and Poly-alpha methylstyrene (PAMS10k) ) have been fabricated by the wire-bar coating technique in ambient conditions (air, light, humidity) and contrasted with the ones prepared by thermally evaporating the organic semiconductor. The transistors fabricated with DB-TTF:PS10k composites show a clear fieldeffect behavior with p-type characteristics, exhibiting charge carriers mobilities in the range of $0.01 \mathrm{~cm}^{2} / \mathrm{Vs}$, fully comparable with the films obtained by thermal evaporation. However, while the thermally evaporated films show poor stability in air, the wire-bar coated composites films and devices are highly reproducible and exhibit lower threshold voltage values. Thus, we demonstrate the suitability of the wire-bar technique for manufacturing large area devices.
\end{abstract}

Keywords: organic field-effect transistors, wire-bar coating, composite materials

\section{INTRODUCTION}

Since the invention of the transistor in 1947 by Bardeen et al. our daily life was transformed to a continuum of electronic devices, where the majority of such devices are based on inorganic semiconductors, led by silicon. ${ }^{1}$ However, a new branch of electronics offers a route to tackle particularly the convoluted fabrication processes that silicon based electronics possess. ${ }^{2}$ The new branch is based on organic compounds and is called Organic Electronics (OE) and today constitutes a whole library of materials mainly based on a vast number of organic semiconductors (OSCs) which have a great potential for the development of a new generation of electronic devices where low-cost, and large area coverage are required. ${ }^{3}$ Indeed, such organic materials are of great importance and interest for cheap electronic applications, as they may often be solution-processed using novel fabrication processes allowing the fabrication of devices such as circuits, displays, and radio-frequency identification devices among many others. ${ }^{4}$ Following this trend, organic field-effect transistors (OFETs) have grown steadily as promising building blocks of low-cost electronic circuitry. OFETs can be a very good alternative to amorphous silicon (a-Si) and can give rise to a novel generation of flexible devices and sensors. The progress in OFETs is particularly due to the advances in material synthesis, theoretical calculations and a development of unconventional yet innovative fabrication techniques to reach low-cost, large-area printed-like electronic devices. ${ }^{5}$ Solution-processed OFETs exhibiting high performance have been reported in the last years. However, the limitations towards applications lie on finding low-cost deposition methods compatible with up-scaling techniques such as roll-to-roll that permit to cover large areas with organic semiconductors. Spin coating and drop casting techniques are widely used to deposit organic semiconductors, but none of them are suitable for up-scaling. Among the unconventional fabrication methods, the convective self-assembly is one widely used for depositing uniform coatings across large areas on a small timescale. ${ }^{6,7}$ One way to achieve a convective self-assembly is using a wire-bar coater ${ }^{8}$ to spread the solution over a substrate provoking the self-assembly of the functional molecules on top of the substrate's surface.

Organic Field-Effect Transistors XIV; and Organic Sensors and Bioelectronics VIII, edited by lain McCulloch, Oana D. Jurchescu, Ioannis Kymissis, Ruth Shinar, Luisa Torsi, Proc. of SPIE Vol. 9568, 95680D

(C) 2015 SPIE · CCC code: $0277-786 X / 15 / \$ 18 \cdot$ doi: $10.1117 / 12.2186521$ 
Organic small molecules are difficult to process as thin films and are often very sensitive upon exposure to ambient conditions. In order to improve this, blending the OSC molecules with polymeric binders has been pursued during the recent years achieving promising results. ${ }^{8,9-14}$ Besides increasing processability and stability, blending the organic semiconductors forming composites also causes a decrease in the fabrication cost due to a reduction of the amount of the active material required, so providing this strategy an additional benefit. ${ }^{8}$ In this study, dibenzo-tetrathiafulvalene (DBTTF) is selected as semiconducting material. This material belongs to the family of tetrathiafulvalenes (TTF) which have shown to exhibit high mobility values in OFETs. DB-TTF is the TTF derivative which has been more studied for the fabrication of OFETs, both as single crystal and thin films. However, despite being relatively quite soluble in organic solvents, thin film OFETs with this material have only been described by thermal evaporation of the organic semiconductor. Here, we study OFETs based on composites of DB-TTF and styrenic insulating polymeric matrix. The active layer can be produced by a solution-based convective deposition technique using a wire-bar coater. We compare their performance with the one achieved by thermally evaporating the OSC.

\section{EXPERIMENTAL METHODS}

The OFETs substrates were made of an $n$-doped silicon $\left(\mathrm{n} \sim 3 \cdot 10^{17} \mathrm{~cm}^{-3}\right)$ as gate and silicon oxide $\left(\mathrm{SiO}_{2}\right)$ layer of $230 \mathrm{~nm}$ as insulator and were purchased from the Fraunhofer IPMS. The source and drain electrodes were made of $30 \mathrm{~nm}$ gold over $10 \mathrm{~nm}$ ITO, which acts as adhesion layer. The electrodes are interdigitated with $10 \mu \mathrm{m}$ of channel length $(L)$ and 10 $\mathrm{mm}$ of width $(W)$. Thermally evaporated devices were fabricated by first cleaning the substrates with acetone and isopropyl alcohol in an ultrasonic bath, and depositing a layer of $50 \mathrm{~nm}$-thick of DB-TTF (Sigma Aldrich) with a evaporation rate of about $0.5 \AA / \mathrm{s}$ on the OFET substrates. All thermally evaporated OFETs were measured in an inert atmosphere inside of a glovebox. Organic thin films from solution of the blends were formed using a K101 K Control Coater from RK PrintCoat Instruments Ltd with standard wire-bar under ambient conditions (air, light, humidity, etc?). Film Deposition was done at $105^{\circ} \mathrm{C}$ temperature controlled by the heated bed on the coating machine. All blends of DBTTF and styrenic matrices (Polystyrene and Poly-alpha methyl styrene all from Sigma-Aldrich) were started from solutions at $1 \% \mathrm{wt}$ in chlorobenzene. All materials have been used as purchased without further purification. The coating speed was adjusted to $1 \mathrm{~cm} / \mathrm{s}$. Coated test chips were left under vacuum at $60^{\circ} \mathrm{C}$ for 2 hours in order to remove residual solvent. All device electrical tests were undertaken at room temperature, in dark and ambient conditions. To test repeatability and reproducibility 40 nominally identical devices were tested. Electrical measurements were performed using a Keithley 2612A sourcemeter with home-made MATLAB software.

\section{RESULTS AND DISCUSSION}

In order to first assess the characteristics of the organic semiconductor, thermally evaporated thin films were fabricated and then electrically characterized. Figure 1(left) depicts the output characteristic of a transistor based on DB-TTF. This device exhibits clear p-type behavior. The OFET has a little series resistance at the injecting electrode due to the small energy offset between the HOMO level of DB-TTF $\left(4.8-4.9 \mathrm{eV}^{15,16}\right)$ and the work function of the electrodes, as shown by the output characteristics at low drain voltages. The field-effect mobility is $2 \cdot 10^{-2} \mathrm{~cm}^{2} / \mathrm{Vs}$. The reproducibility of the mobility value of the OFETs based on DB-TTF is very high. In this work, an average mobility value of $0.02 \pm 0.008$ $\mathrm{cm}^{2} /$ Vs was extracted from measuring 24 devices.

The electronic characteristics of DB-TTF as organic semiconductor are very appealing. However, due to its low HOMO level DB-TTF is easily doped when exposed to ambient conditions. This effect has an influence on their device electrical characteristics, mainly on the switch-on voltage $\left(V_{S O}\right)$. Figure 1(right) depicts the semilog-transfer characteristic measured with time within a glove box in nitrogen atmosphere with low oxygen and humidity concentrations $\left(\mathrm{O}_{2}<2\right.$ ppm and $\mathrm{H}_{2} \mathrm{O}<3 \mathrm{ppm}$ ). The measurements interval is 12 minutes. A shift in the $V_{S O}$ from $-1 \mathrm{~V}$ to $3 \mathrm{~V}$ in only 36 minutes was observed, which is remarkable considering the atmospheric conditions where the device is placed and reflects the fact that this material is prone to oxidation. Further, it is also worth mentioning that, contrary to the mobility values, we found that the switch-on voltage for thermally evaporated DB-TTF OFETs is not very reproducible. In this work, the 
range of $V_{S O}$ found was from -1 $\mathrm{V}$ to $+45 \mathrm{~V}$. This phenomenon has also been reported in previous works also focused on OFETs based on DB-TTF deposited by thermal evaporation. ${ }^{16-18}$ Noda et al. ascribes the fact that OFETs tend to be in the 'on' state at $V_{G S}=0 \mathrm{~V}$ due to the energy difference between the HOMO level of DB-TTF and the work function of gold. Further, the variation of $V_{S O}$ has been attributed to the morphology of the first molecular monolayers of DB-TTF. ${ }^{18}$ We found that the functionalisation of the $\mathrm{SiO}_{2}$ dielectric with an OTS (octadecyltrichlorosilane) monoalyer did not help to improve the large variation of $V_{S O}$ values obtained in the devices. Thus, we can summarize that the reproducibility achieved with the fabricated DB-TTF OFETs by thermally evaporation is very high in terms of mobility values but, in contrast, the devices show a large spread in the switch-on voltage values. Often, such $V$ so values are highly positive and, always, they shift to more positive values with time, even at ppm levels of oxygen and water. This can be explained by the high impact that the first deposited DB-TTF monolayers might have on the device performance and also the fact that this material gets easily doped. The obtained mobility values and performance for thermally evaporated films match the reported values found in the literature. ${ }^{17,19}$
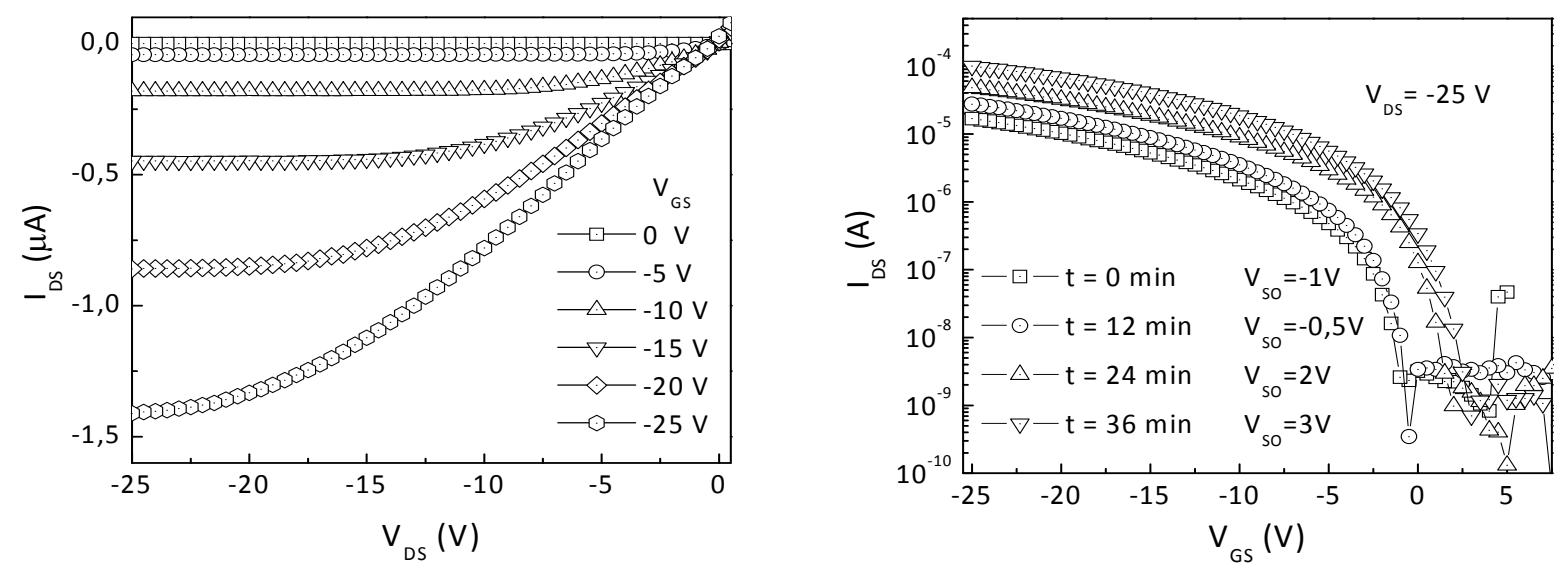

Figure 1. Electrical characteristics for a typical OFET fabricated by thermal evaporation and measured in an inert atmosphere $\left(\mathrm{O}_{2}<2\right.$ ppm and $\left.\mathrm{H}_{2} \mathrm{O}<3 \mathrm{ppm}\right)$. Left. Output characteristics. Right. Semi-log-transfer and evolution of the switch-on voltage with time under inert conditions.

a)

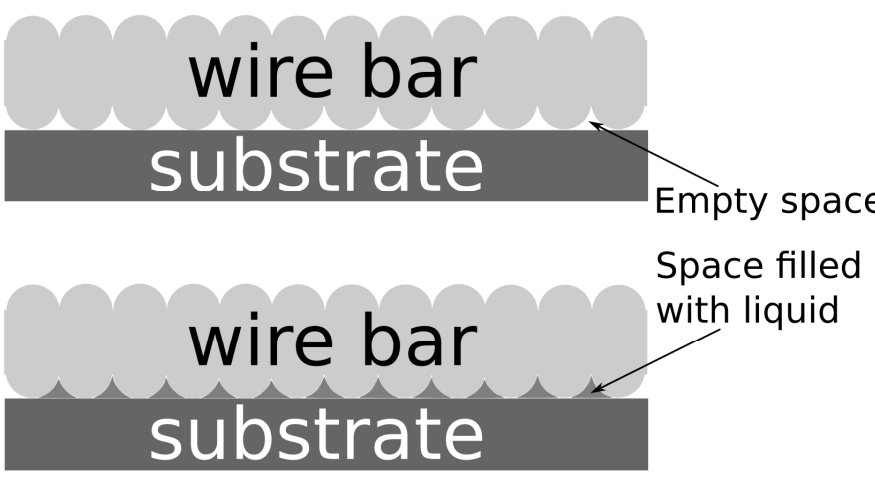

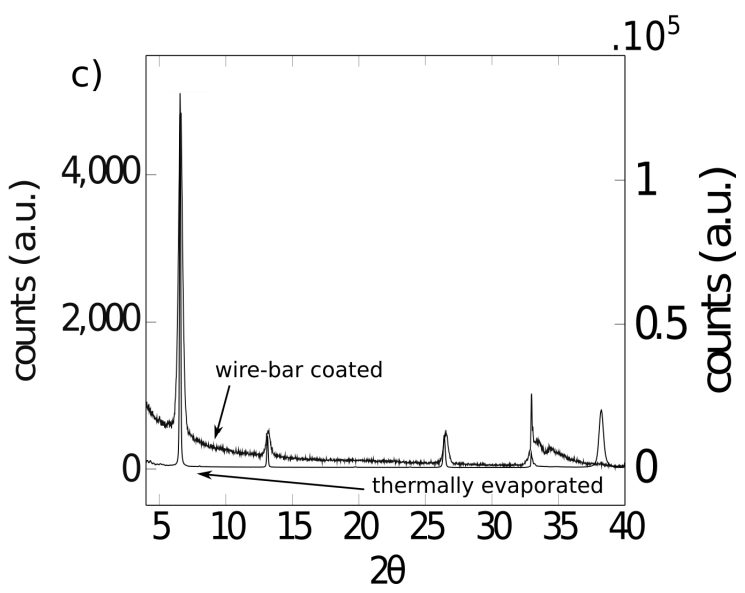

$2 \theta$

Figure 2. a) and b) Schematic representation of the wire-bar coating and c) X-ray diffraction pattern of the DB-TTF films produced by thermal evaporation and by wire-bar coating. 
OFETs obtained by thermal evaporation are of great interest for theory and modeling due to the high purity of the organic semiconductor. High control of the deposited layers at molecular rates - $\AA / \mathrm{s}$ - permits to understand the film formation and its influence on the electrical characteristics. However, solution-based techniques are more suitable for large area production at low-cost. Thus, nowadays it is imperative to come up with a procedure able to deposit the organic semiconductors, ideally under ambient conditions and at high speeds, to reach industrial applications. In this endeavor, thin films of several blends of DB-TTF and styrenic matrices were deposited on $\mathrm{Si}_{2} / \mathrm{SiO}_{2}$ employing a wire-bar coating technique, which is schematically represented in Figure $2 a$ and $b$. In this method, a wire-bar is allowed to smoothly contact the surface and the spaces between the wires are filled with the solution to be coated. The fluid is sheared by the wire-bar, movement allowing the formation of a crystalline thin film by convective self-assembly. ${ }^{7,820-21}$ Figure 2c depicts a comparison of the X-ray diffraction patterns found for both a thermally evaporated thin film and a typical film fabricated by the wire-bar coating method. One can clearly observe that both diffraction patterns agree in more than three peaks. Also the typical peak of the silicon substrate which is shining through the $\mathrm{SiO}_{2}$ is clearly visible. The crystallographic DB-TTF phase present in the films has been identified to correspond to the gamma phase of DBTTF which was previously reported by Brillante $e t a l .{ }^{22}$ This phase is the most common one formed on a surface thin film since is the kinetically most favorable phase.

The styrenic matrices employed were polystyrene (PS) for Gel Permeation Chromatography (GPC) standard with a molecular weight of $10000 \mathrm{~g} / \mathrm{mol}$ (10k) and poly(alpha-methyl styrene) (PAMS) with a molecular weight of 10000 $\mathrm{g} / \mathrm{mol}$ (10k). Such polymers were blended with DB-TTF with blend ratios of 50:50, 60:40 and 70:30 of semiconductor:styrenic matrix, respectively. Nonetheless, the best performance was realized with the blends 50:50 ratio, since it was observed that lowering the proportion of the organic semiconductor below $50 \%$ the device mobility abruptly decreased by around 2 orders of magnitude.
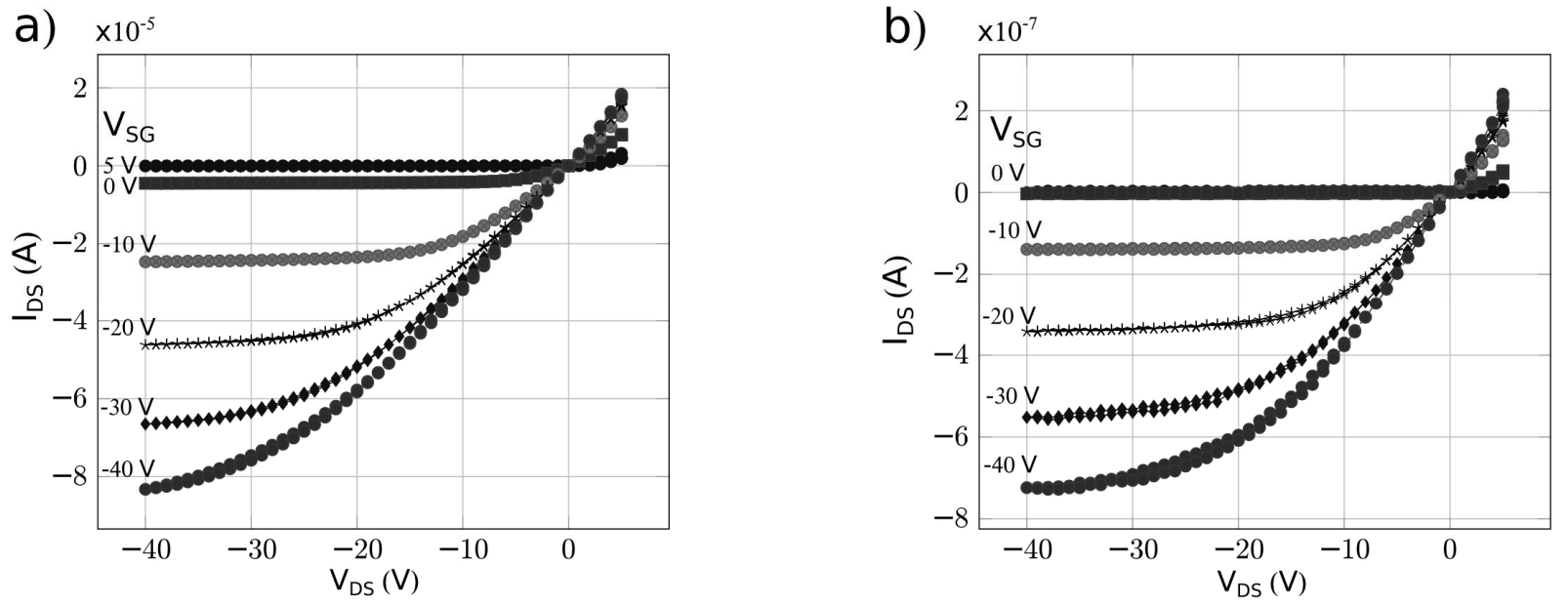

Figure 3. Output characteristics for DB-TTF composites OFETs fabricated entirely in air by the wire-bar coating technique for (a) composite based on PS10k and (b) composite based on PAMS10k.

Figure 3 depicts the output characteristics for two the different styrenic matrices PS10k and PAMS10k both at the same ratio semiconductor and styrenic matrix which is 50:50 and employing the same transistor architecture maintaining the same ratio width-length equal to 1000. In both cases, one can observe a neat p-type behavior. However, it is clearly noticeable that the two devices reveal two orders of magnitude difference in the drain-source current, being the device based on PS10k the one exhibiting higher current values. This difference can be probably ascribed to the difference of chemical composition of the polymeric matrix that may produce a difference in the segregation of the DB-TTF semiconductor. Mobility wise the maximum values found were $0.020 \mathrm{~cm}^{2} / \mathrm{Vs}$ for PS10k based composites and 0.002 
$\mathrm{cm}^{2} / \mathrm{Vs}$ for PAMS10k, while the threshold voltages were $5.2 \mathrm{~V}$ and $-7.2 \mathrm{~V}$ respectively. These values suggest that an enhanced stability has been achieved since all processing steps as well as the electrical characterization of these devices were carried out in air. Such high stability can be tentatively attributed to a vertical phase separation provoking that the active layer or hole-transporting layer is buried under a protective styrenic matrix. Therefore, by mixing the DB-TTF semiconductor with the polymer, solution processability and device stability has been achieved.

\section{CONCLUSIONS}

OFETs based on thin films of the most well-known tetrathiafulvalene (TTF) organic semiconductor, that is DB-TTF, have been investigated by thermal evaporation as well as solution processing using a standard wire-bar coating technique. The thermally evaporated thin films of DB-TTF showed reproducible mobility values of around $0.02 \mathrm{~cm}^{2} / \mathrm{Vs}$ when measured under an inert atmosphere. However, the $\mathrm{V}_{\mathrm{SO}}$ of such devices exhibited a large shift of towards more positive voltages, which is related to the high sensitivity of DB-TTF to oxidation even under very low concentration of water and oxygen. On the other hand, for achieving solution processability, DB-TTF was blended with insulating styrenic matrices employing a range of composition ratios. These devices were entirely fabricated and measured in air, exhibiting high air-stability. The most optimum devices showed mobilities in the order of $10^{-2} \mathrm{~cm}^{2} / \mathrm{Vs}$, thus matching the values obtained by thermal evaporation. Therefore, we demonstrate that the wire coating technique combined with the blending of the organic semiconductor provides a promising route towards the realization of low-cost and stable devices.

\section{REFERENCES}

[1] Mas-Torrent, M. \& Rovira, C. Novel small molecules for organic field-effect transistors: towards processability and high performance. Chem. Soc. Rev. 37, 827-838 (2008).

[2] Bardeen, S. J. \& Shockley, W. Organic thin film transistors. Mater. Today 20-27 (2004).

[3] Facchetti, A. Semiconductors for organic transistors. Mater. Today 10, 28-37 (2007).

[4] Reese, C. \& Bao, Z. Organic single-crystal field-effect transistors. Mater. Today 10, 20-27 (2007).

[5] Wen, Y., Liu, Y., Guo, Y., Yu, G. \& Hu, W. Experimental Techniques for the Fabrication and Characterization of Organic Thin Films for Field-Effect Transistors. Chem. Rev. 111, 3358-3406 (2011).

[6] Chen, K., Stoianov, S. V, Bangerter, J. \& Robinson, H. D. Restricted meniscus convective selfassembly. J. Colloid Interface Sci. 344, 315-320 (2010).

[7] Prevo, B. G., Kuncicky, D. M. \& Velev, O. D. Engineered deposition of coatings from nano- and micro-particles: A brief review of convective assembly at high volume fraction. Colloids Surfaces A Physicochem. Eng. Asp. 311, 2-10 (2007).

[8] Murphy, C. E. et al. Wire-bar coating of semiconducting polythiophene/insulating polyethylene blend thin films for organic transistors. J. Appl. Phys. 110, 93523 (2011).

[9] Arias, A., Endicott, F. \& Street, R. Surface-Induced Self-Encapsulation of Polymer Thin-Film Transistors. Adv. Mater. 18, 2900-2904 (2006).

[10] Arias, A. C., MacKenzie, J. D., McCulloch, I., Rivnay, J. \& Salleo, A. Materials and applications for large area electronics: solution-based approaches. Chem. Rev. 110, 3-24 (2010). 
[11] Hamilton, B. R. et al. High-Performance Polymer-Small Molecule Blend Organic Transistors. Adv. Mater. 21, 1166-1171 (2009).

[12] Hwang, D. K., Fuentes-Hernandez, C., Kim, J. B., Potscavage, W. J. \& Kippelen, B. Flexible and stable solution-processed organic field-effect transistors. Org. Electron. 12, 1108-1113 (2011). [13] Madec, M.-B., Morrison, J. J., Sanchez-Romaguera, V., Turner, M. L. \& Yeates, S. G. Organic field effect transistors from ambient solution processed poly(triarylamine)-insulator blends. J. Mater. Chem. 19, 6750-6755 (2009).

[14] Ferenczi, T. A. M. et al. Organic Semiconductor : Insulator Polymer Ternary Blends for Photovoltaics. Adv. Mater. 23, 4093-4097 (2011).

[15] Mas-Torrent, M. et al. Single-crystal organic field-effect transistors based on dibenzotetrathiafulvalene. Appl. Phys. Lett. 86, 12110 (2005).

[16] Li, H., Zheng, R. \& Shi, Q. Theoretical study on charge carrier mobilities of tetrathiafulvalene derivatives. Phys. Chem. Chem. Phys. 13, 5642-5650 (2011).

[17] Noda, B. et al. Crystal structures and transistor properties of phenyl-substituted tetrathiafulvalene derivatives. Nanotechnology 18, 424009 (2007).

[18] Naraso et al. High-Performance Organic Field-Effect Transistors Based on pi-Extended Tetrathiafulvalene Derivatives. J. Am. Chem. Soc. 127, 10142-10143 (2005).

[19] Noda, B. et al. Organic Field-effect Transistor Based on Biphenyl Substituted TTF. Chem. Lett. 34, 392-393 (2005).

[20] Chen, K., Stoianov, S. V, Bangerter, J. \& Robinson, H. D. Restricted meniscus convective selfassembly. J. Colloid Interface Sci. 344, 315-20 (2010).

[21] Prevo, B. G. \& Velev, O. D. Controlled, Rapid Deposition of Structured Coatings from Microand Nanoparticle Suspensions. Langmuir 20, 2099-2107 (2004).

[22] Brillante, A. et al. The four polymorphic modifications of the semiconductor dibenzotetrathiafulvalene. Cryst. Eng. Comm 10, 1899-1909 (2008). 